\title{
A New Direction in Al \\ Toward a Computational Theory of Perceptions
}

\author{
Lotfi A. Zadeh
}

- Humans have a remarkable capability to perform a wide variety of physical and mental tasks without any measurements and any computations. Familiar examples are parking a car, driving in city traffic, playing golf, cooking a meal, and summarizing a story. In performing such tasks, humans use perceptions of time, direction, speed, shape, possibility, likelihood, truth, and other attributes of physical and mental objects. Reflecting the bounded ability of the human brain to resolve detail, perceptions are intrinsically imprecise. In more concrete terms, perceptions are f-granular, meaning that (1) the boundaries of perceived classes are unsharp and (2) the values of attributes are granulated, with a granule being a clump of values (points, objects) drawn together by indistinguishability, similarity, proximity, and function. For example, the granules of age might be labeled very young, young, middle aged, old, very old, and so on.

F-granularity of perceptions puts them well beyond the reach of traditional methods of analysis based on predicate logic or probability theory. The computational theory of perceptions (CTP), which is outlined in this article, adds to the armamentarium of $\mathrm{Al}$ a capability to compute and reason with perception-based information. The point of departure in CTP is the assumption that perceptions are described by propositions drawn from a natural language; for example, it is unlikely that there will be a significant increase in the price of oil in the near future.

In CTP, a proposition, $p$, is viewed as an answer to a question, and the meaning of $p$ is represented as a generalized constraint. To compute with perceptions, their descriptors are translated into what is called the generalized constraint language (GCL). Then, goal-directed constraint propagation is utilized to answer a given query. A concept that plays a key role in CTP is that of precisiated natural Ianguage (PNL).

The computational theory of perceptions suggests a new direction in $\mathrm{Al}-$ a direction that might enhance the ability of Al to deal with real-world problems in which decision-relevant information is a mixture of measurements and perceptions. What is not widely recognized is that many important problems in Al fall into this category.

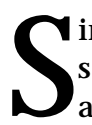
nceits inception in the early fifties, Al has cored a number of major successes, among them the defeat of Gary Kasparov by DEEP BLUE. However, what we also see is that alongside the brilliant achievements lie areas in which progress has been slow and difficult to realize. In such areas, problems do not lend themselves to precise formulation, and the underlying modes of reasoning are approximate rather than exact. A case in point is the problem of summarization-a problem that is orders of magnitude more complex than the problem of machine translation. Although substantial progress has been realized (Mani and Maybury 1999), we are still far from being able to construct programs that are capable of summarizing a nonstereotypical story or providing a synopsis of a book.

Why is it that major successes have been achieved in some areas but not in others? A thesis that I should like to put on the table is that progress has been, and continues to be, slow in those areas where a methodology is needed in which the objects of computation are perceptions-perception s of time, distance, form, direction, color, shape, truth, likelihood, intent, and other attributes of physical and mental objects.

Humans have a remarkable capability to perform a wide variety of physical and mental tasks without any measurements and any computations. Everyday examples of such tasks are parking a car, driving in city traffic, playing 


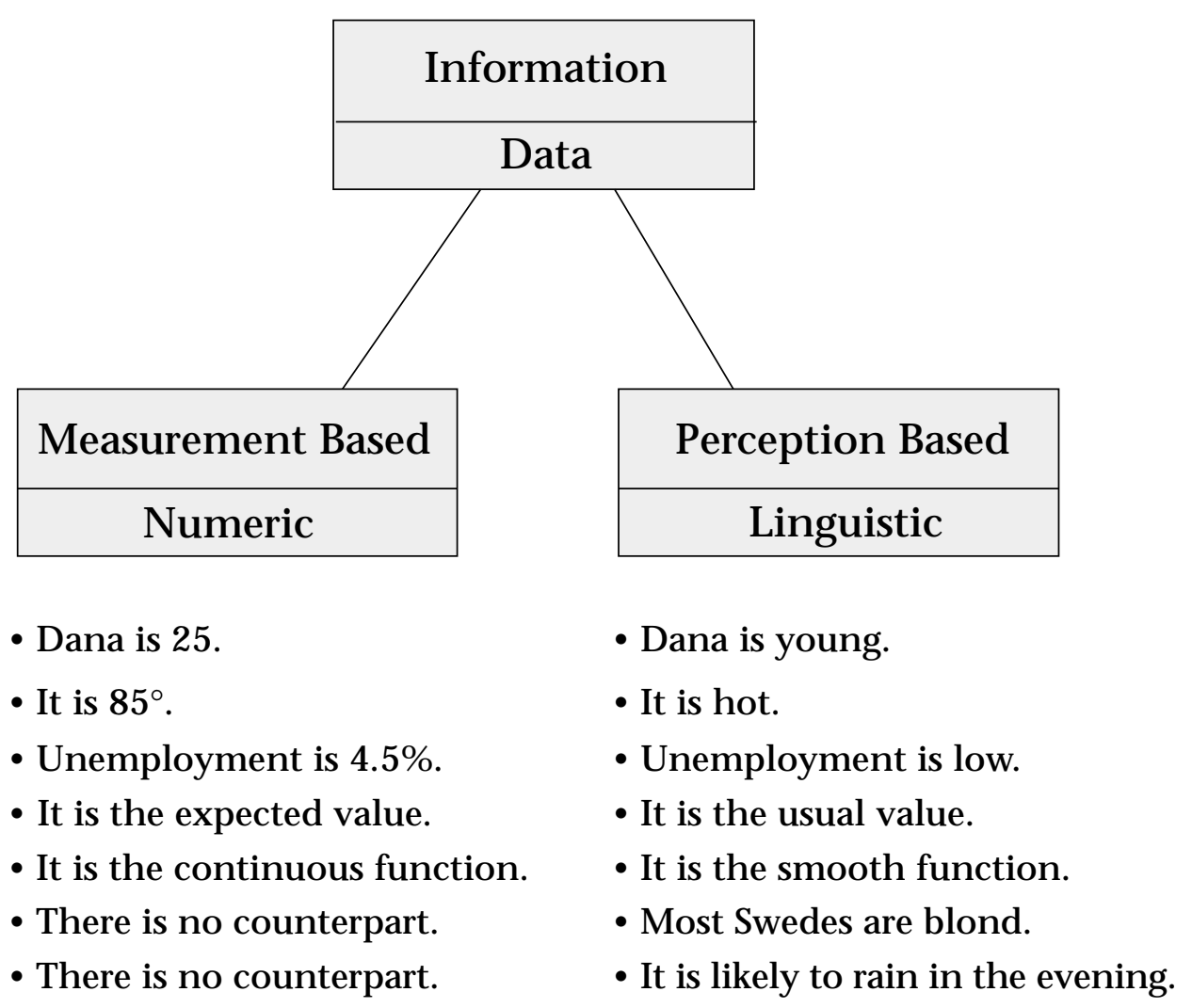

Figure 1. Structure of Information.

Examples of measurement-based and perception-based information.

golf, cooking a meal, and summarizing a story. In performing such tasks, for example, driving in city traffic, humans base whatever decisions have to be made on information that, for the most part, is perception, rather than measurement, based. The computational theory of perceptions (CTP), which is outlined in this article, is inspired by the remarkable human capability to operate on, and reason with, perception-based information.

An essential difference between measurements and perceptions is that in general, measurements are crisp, whereas perceptions are fuzzy (figure 1). Furthermore, the finite ability of sensory organs to resolve detail necessitates a partitioning of objects (points) into granules, with a granul ebeing a clump of objects (points) drawn together by indistinguishability, similarity, proximity, or function. Thus, perceptions, in general, are both fuzzy and granular or, for short, f-granular. For example, a perception of age can be described as very young, young, middle aged, old, and very old, with very young, young, and so on, constituting the granules of the variable age (figure 2 ). In this perspective, natural languages can be viewed as systems whose primary function is to describe perceptions.

Information granulation plays key roles in both human and machine intelligence. Modes of information granulation in which the granules are crisp, that is, c-granular (figure 3), play important roles in a wide variety of methods, approaches, and techniques. Among them are interval analysis, quantization, rough-set theory, diakoptics, divide and conquer, DempsterShafer theory, machine learning from examples, chunking, qualitative process theory, qualitative reasoning, decision trees, semantic networks, analog-to-digital conversion, constraint programming, Prolog, and cluster analysis.

Important though it is, crisp information granulation has a major blind spot. More specifically, it fails to reflect the fact that in much, perhaps most, of human reasoning and concept formation, the granules are fuzzy ( $f$ granular) rather than crisp. In the case of a 

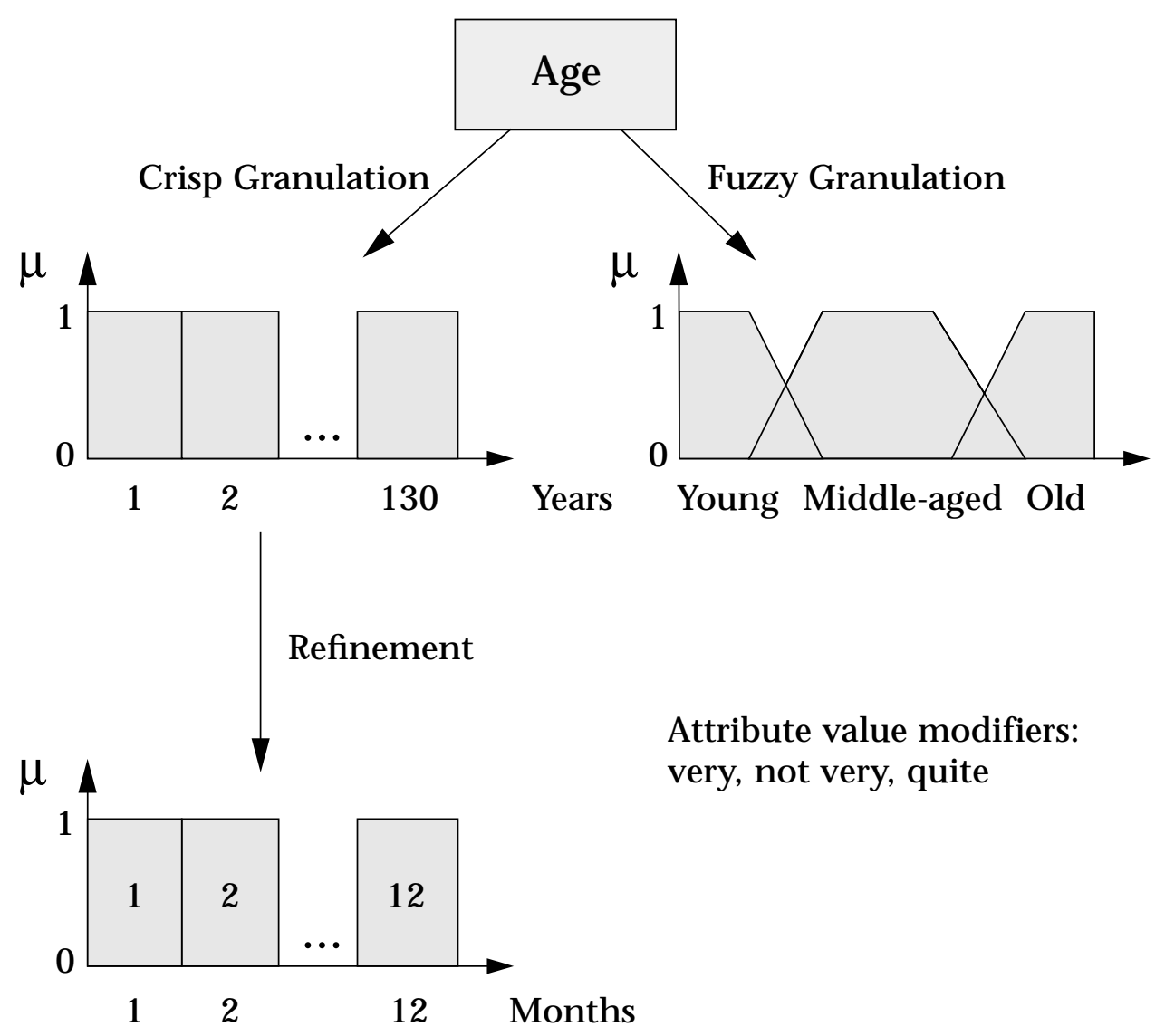

Attribute value modifiers: very, not very, quite

Figure 2. F-Granulation and C-Granulation of Age.

human body, for example, the granules are fuzzy in the sense that the boundaries of the head, neck, arms, legs, and so on, are not sharply defined. Furthermore, the granules are associated with fuzzy attributes, for example, length, color, and texture in the case of hair. In turn, fuzzy attributes can have fuzzy values; for example, in the case of the fuzzy attribute length (hair), the fuzzy values could be long, short, very long, and so on. The fuzziness of granules, their attributes, and their values is characteristic of the ways in which human concepts are formed, organized, and manipulated (Zadeh 1997; Dubois and Prade 1996).

There is an enormous literature on perceptions, spanning psychology, philosophy, linguistics, neuroscience, cognitive science, system theory, and other fields (Barsalou 1999; Vallee 1995). However, what is not in existence is a theory in which perceptions are objects of computation, as they are in CTP.

In the computational theory of perceptions, the point of departure is not, in general, a collection of perceptions; rather, it is a collection of descriptions of perceptions expressed in a natural language. Consider the following examples: (1) Dana is young, (2) Dana is much younger than her husband, (3) Michelle has long hair, (4) most Swedes are tall, (5) overeating causes obesity, (6) usually Robert returns from work at about $6 \mathrm{pm}$, and (7) it is very unlikely that there will be a significant increase in the price of oil in the near future. Thus, a basic assumption in CTP is that perceptions are represented as propositions in a natural Ianguage.

The principal aim of CTP is the development of a machinery for computing and reasoning with perceptions. A simple example of reasoning with perceptions is the following: Assume that we start with the antecedent perceptions that "most young men are healthy" and that "Robert is a young man," where most is a perceptual (fuzzy) quantifier that is defined by its 


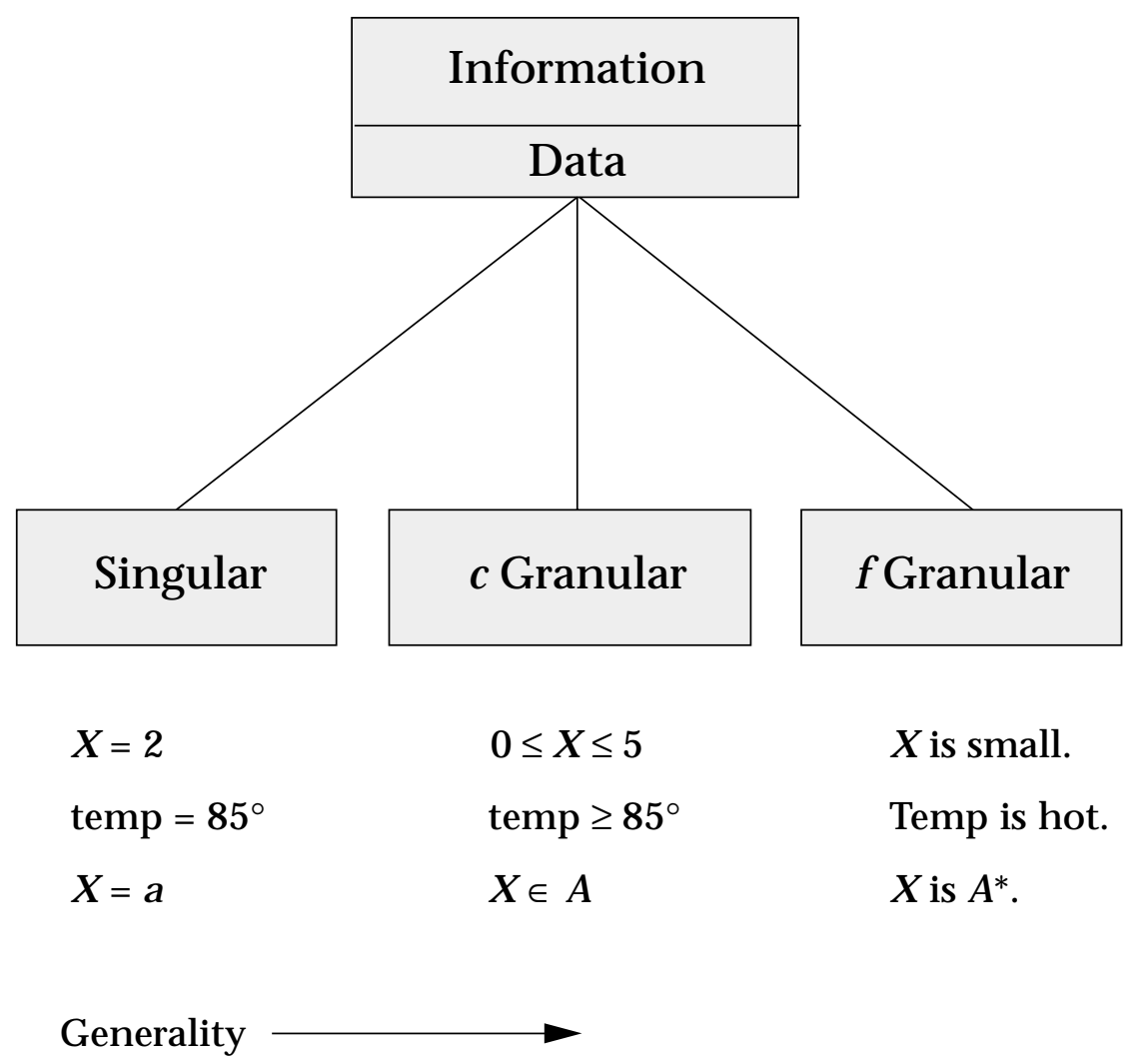

Figure 3. Classification and Successive Generalization of Modes of Granulation: Singular, C-Granular, and F-Granular.

membership function (figure 4). Then, it can be shown that the consequent perception can be expressed as "it is likely that Robert is healthy," where likely is a fuzzy probability whose membership function coincides with that of most. Note that the result of computation in this case is in agreement with intuition.

Within Al, there are methodologies, prominent among which are qualitative reasoning and qualitative process theory (QPT) (Sun 1994; Raiman 1991; Davis 1990; de Kleer and Bobrow 1984; Forbus 1984; Kuipers 1984), that provide effective tools for dealing with interval-valued types of perception-based information. In relation to these methodologies, the computational theory of perceptions is complementary rather than competitive. More concretely, a basic difference between qualitative reasoning and CTP is that in qualitative reasoning, granulation is crisp, whereas in CTP, granulation is fuzzy. Because fuzzy granulation subsumes crisp granulation, CTP, in principle, has a higher level of general ity than qual itative reasoning. However, in comparing the two the- ories, it is standard practice to exclude the less general theory from the more general theory, leaving a theory that complements the less general theory. Thus, in this sense, for example, linear system theory and nonlinear system theory are complementary. The same applies to the complementarity of qualitative reasoning and CTP. In more specific terms, the following examples illustrate the types of problems that fall within the scope of the computational theory of perceptions.

First is perception-based function modeling. Perception of a function can be described as a collection of linguistic if-then rules, with each rule describing a fuzzy granule. For example, in the case of the function, $f$, shown in figure 5, a description of a perception of $f$ can be expressed as

If $X$ is small, then $Y$ is small.

If $X$ is medium, then $Y$ is large.

If $X$ is large, then $Y$ is small.

Given a perception-based description of a function, $f$, various standard questions can be raised; for example, what is the maximum val- 


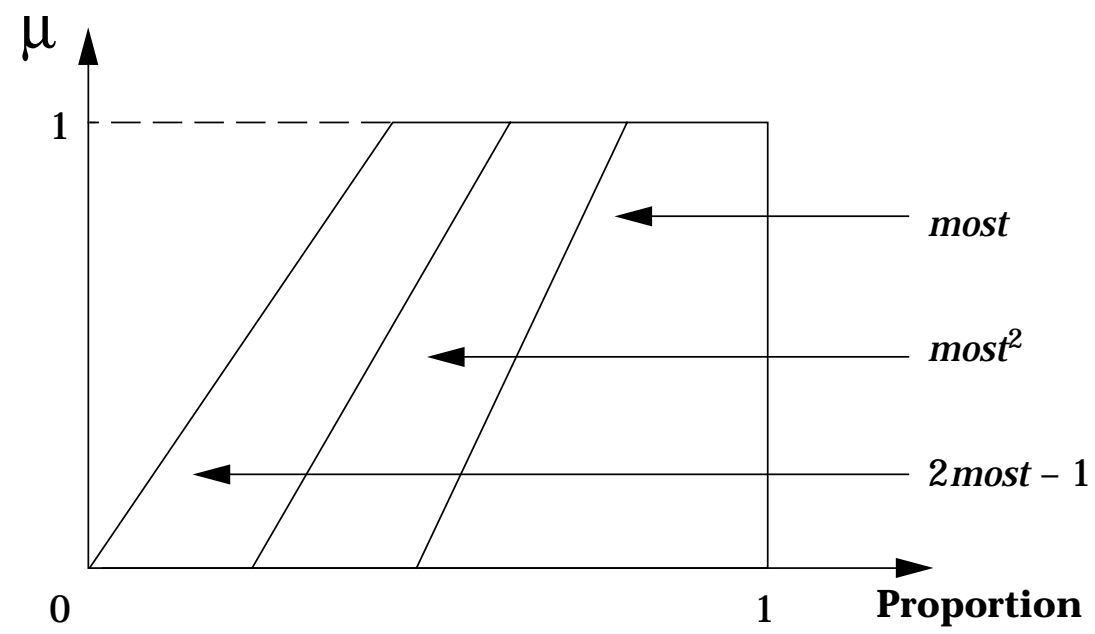

most ${ }^{2} \geq 2$ most -1

$x^{2} \geq 2 x-1$

Figure 4. M embership Functions of "M ost" and Related Perceptual (Fuzzy) Quantifiers.

ue of $f$ ? Such questions are representative of perception-based computations in which the objects of computation are perception-based models of functions (Zadeh 1999).

Second is perception-based system modeling. A system, $\mathrm{S}$, is assumed to be associated with temporal sequences of input $X_{1}, X_{2}, \ldots$; output $Y_{1}$, $Y_{2}, \ldots$; and states $S_{1}, S_{2}, \ldots . S$ is defined by the state-transition function $f$

$$
\mathrm{S}_{\mathrm{t}+1}=\mathrm{f}\left(\mathrm{S}_{\mathrm{t}}, \mathrm{X}_{\mathrm{t}}\right)
$$

and the output function $g$

$$
Y_{t}=g\left(S_{t}, X_{t}\right) \text {. }
$$

In perception-based system modeling, the input, the output, and the states are assumed to be perceptions, as are the state-transition function, $f$, and the output function, $g$. Thus, a perception-based model of $\mathrm{S}$ would consist of a collection of linguistic if-then rules of the generic form "if $X_{t}$ is $A_{t}$ and $S_{t}$ is $B_{t}$, then $S_{t+1}$ is $C_{t}$, and $Y_{t}$ is $D_{t}, t=1,2, \ldots$, where $A_{t}, B_{t}, C_{t}$, $D_{t}, t=1,2, \ldots$, are $f$-granular perceptions of the values of $X_{t}, S_{t}, S_{t+1}$, and $Y_{t}$ (figure 6).

It is important to note that much of human decision making in everyday settings involves a knowledge of perception-based models of various systems, for example, when we drive a car, balance a pole, and play golf. It is of interest to note that perception-based system mod- eling underlies what we normally view as commonsense and qualitative reasoning (Struss 1990; Mavrovouniotis and Stephanopoulos 1987; Forbus 1984; Kuipers 1984; Zadeh 1973; McCarthy and Hayes 1969).

Third is perception-based time-series analysis. In dealing with time series, the usual assumption is that their elements are numbers. In perception-based time-series analysis, the assumption is that the elements are perceptions, for example, small, medium, and large (figure 7). A question that can be raised is, What is theaverage value of a given time series? An important problem in perception-based time-series analysis is forecasting in the context of time series that are sequences of perceptions rather than measurements.

Fourth is the solution of perception-based equations. An example would be solving a system of equations with perception-based coefficients; for example,

$$
\text { small } \cdot x+\text { large } \cdot y=\text { medium }
$$$$
\text { medium } \cdot x+\text { small } \cdot y=\text { large }
$$

Fifth is computation with perception-based probabilities. In probability theory, the usual assumption is that probabilities are numeric. In reality, most probabilities, and especially subjective probabilities, are perception based. For 


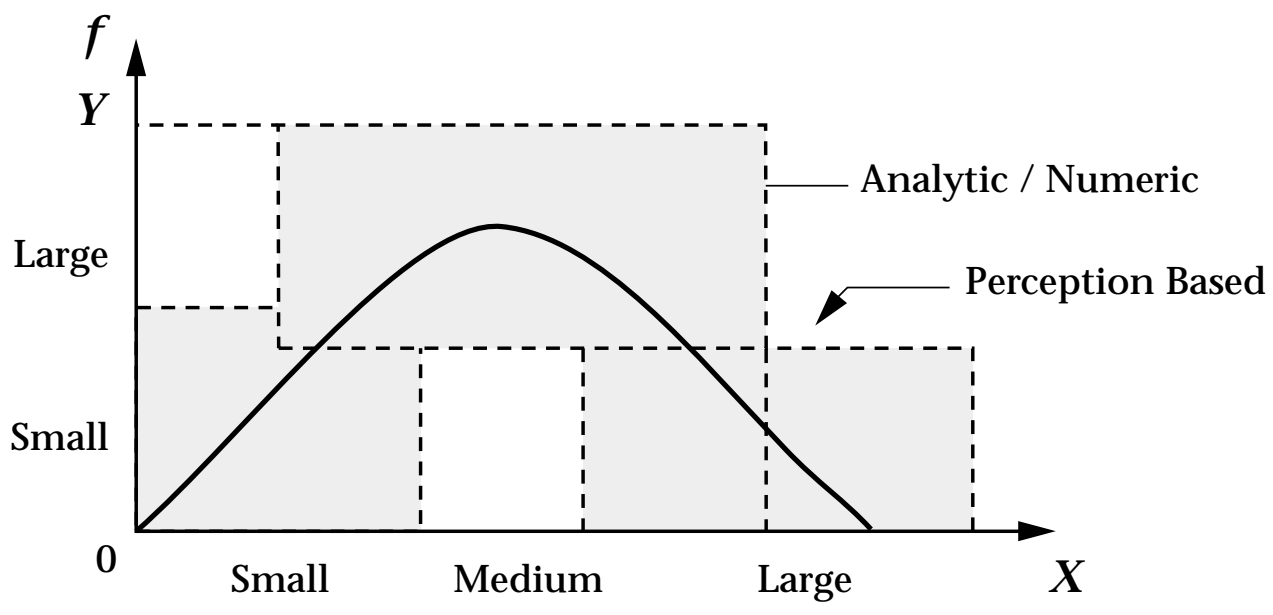

$f:$ If $X$ is small, then $Y$ is small.

If $X$ is medium, then $Y$ is large.

If $X$ is large, then $Y$ is small.

Figure 5. Perception-Based Function Representation as a Collection of Linguistic If-Then Rules.

The granules in the $(X, Y)$ space are Cartesian products of the granules in the $X$ space and $Y$ space.

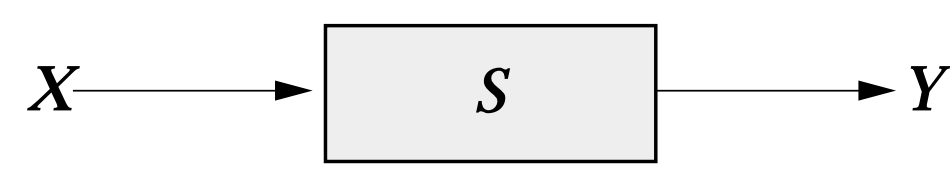

Input: $X_{1}, X_{2}, \ldots$

Output: $Y_{1}, Y_{2}, \ldots$

States: $\mathrm{S}_{1}, \mathrm{~S}_{2}, \ldots$

State-Transition Function: $S_{t+1}=f\left(S_{t}, X_{t}\right), t=1,2, \ldots$

Output Function: $Y_{t}=g\left(S_{t}, X_{t}\right)$

If $S_{t}$ is small and $X_{t}$ is small, then $S_{t+1}$ is small.

If $S_{t}$ is small and $X_{t}$ is medium, then $S_{t+1}$ is large.

Figure 6. Perception-Based System M odeling.

Transition and output functions are represented as collections of linguistic if-then rules. example, I might have the perception that most balls, in a box that contains balls of various sizes, are large. In this case, a perception of the probability that a ball drawn at random is large might be described as most, where most is interpreted as a fuzzy proportion (figure 2).

A less simple version of this example is the following: Assume that a box contains balls of various sizes and that my perceptions are (1) there are about 20 balls in the box; (2) most are large, and (3) a few are small. The question is, What is the probability that a ball drawn at random is neither large nor small?

As a further example of perception-based probabilistic reasoning, assume that I know that usually Robert returns from work at about 6 PM. The question is, What is the probability that Robert is home at 6:30 PM? Another question is, What is the earliest time that the probability that Robert is home is high?

As was stated at an earlier point, existing methods for dealing with perception-based information are effective when perceptions are interval valued or, more generally, c-granular. However, in most everyday settings, perception-based information is, as a rule, f-granular rather than c-granular. In this perspective, the importance of the computational theory of 
perceptions derives in large measure from the fact that it provides a capability to process perception-based information that is not constrained to be c-granular.

To be able to reason with perceptions, it is necessary to have a mean s of representing their meaning in a form that lends itself to computation. The expressive power of conventional predicate-logic-based meaning representation languages is insufficient for this purpose. This applies to very simple propositions such as "most balls are large" and "usually Robert returns from work at about 6 PM" and even more so to a proposition such as "it is very unlikely that there will be a significant increase in the price of oil in the near future."

In the computational theory of perceptions, meaning representation is based on the use of what is referred to as constraint-centered semantics of natural languages (CSNL). CSNL is a key part of CTP. The basic concepts underlying CSNL are outlined in the following section.

\section{Constraint-Centered Semantics of Natural Languages}

The point of departure in CSNL is a set of four basic assumptions.

First, a proposition, $p$, is an answer to a question, q. In general, q is implicit rather than explicit in $\mathrm{p}$.

Second, the meaning of $p$ is a generalized constraint on a variable. In general, both the variable and the constraint to which it is subjected are implicit in $p$. The canonical form of $p, C F(p)$, places in evidence the constrained variable and the constraining relation.

Third, a proposition, $\mathrm{p}$, is viewed as a carrier of information. The canonical form of $p$ defines the information that $p$ carries.

Fourth, in CTP, reasoning is viewed as a form of computation. Computation with perceptions is based on propagation of generalized constraints from premises (antecedent propositions) to conclusions (consequent propositions).

In one form or another, manipulation of constraints plays a central role in a wide variety of methods and techniques, among which are mathematical programming, constraint programming, logic programming, and qualitative reasoning. However, in these methods and techniques, the usual assumption is that a constraint on a variable $X$ is expressible as $X \in A$, where $A$ is a crisp set, for example, $a \leq X \leq b$. In other words, conventional constraints are possibilistic in the sense that what they constrain are the possible values of variables.

The problem is that natural languages are

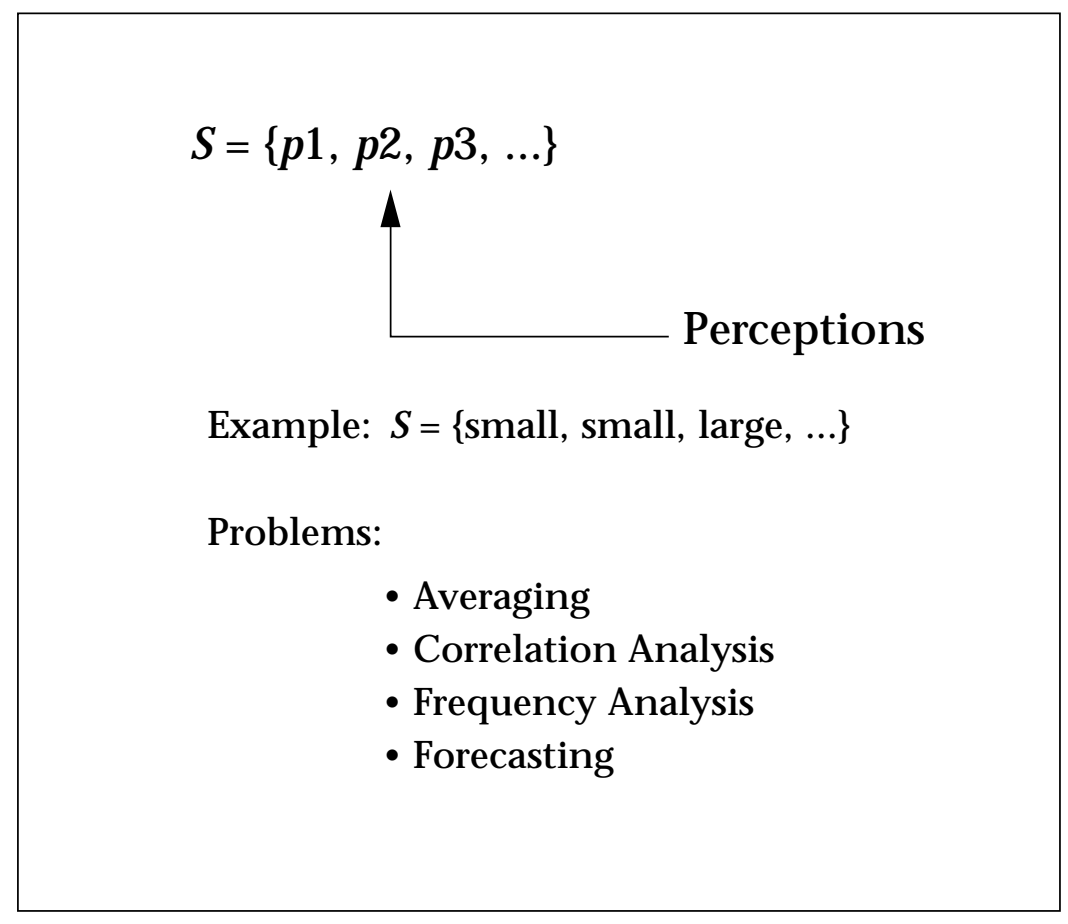

Figure 7. Perception-Valued Time-Series Analysis.

much too rich to fit the Procrustean bed of conventional constraints. For, example, thesign in a hotel, "checkout time is 11 AM," constrains the checkout time. However, can it be represented as "checkout time $=11 \mathrm{Am}$ " or as some other straightforward variation? Clearly, "checkout time is 11 AM" does not have a simple representation.

If our goal is to represent the meaning of a proposition drawn from a natural language as a constraint on a variable, then what is needed is a variety of constraints of different types-a variety that includes the standard constraint as a special case. This is what underlies the concept of a generalized constraint (Zadeh 1986) in CSNL.

A generalized constraint is represented as $X$ isr $R$, where isr, pronounced "ezar," is a variable copula that defines the way in which $R$ constrains X. More specifically, the role of $R$ in relation to $X$ is defined by the value of the discrete indexing variable $r$. The values of $r$ and their interpretations are defined in figure 8.

As an illustration, when $r=e$, the constraint is an equality constraint and is abbreviated to $=$ When $r$ takes the value $d$, the constraint is disjunctive (possibilistic), and isd, abbreviated to is, leads to the expression $X$ is $R$, in which $R$ is a fuzzy relation that constrains $X$ by playing the role of the possibility distribution of $X$ (Zadeh 1997; Lano 1991; Novak 1991).

As alluded to already, the key idea underlying CSNL is that the meaning of a proposition, 


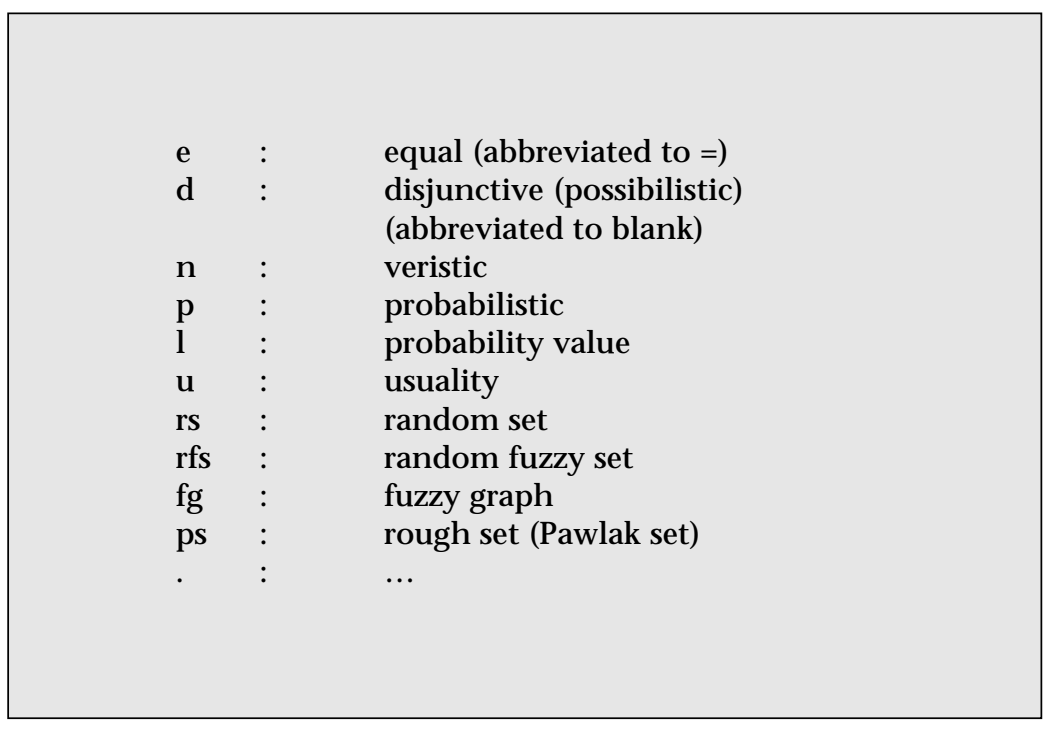

Figure 8. Interpretations of Values of Indexing Variable, r.

$p$, can be represented as a generalized constraint on a variable. Schematically, this is represented as

$$
\mathrm{p} \underset{\text { explicitation }}{\rightarrow} \mathrm{X} \text { isr } \mathrm{R}
$$

with the understanding that the target language of translation is the language of generalized constraints, that is, GCL. Thus, translation is viewed as explicitation of the constrained variable, $X$; the defining indexing variable, $r$; and the constraining relation, $R$. In general, $X$, $r$, and $R$ are implicit rather than explicit in $p$. Furthermore, $X, r$, and $R$ depend on the question to which $p$ is an answer. Thus, explicitation makes explicit what is implicit in $p$.

As a simple example consider the proposition

p: Mary is young.

In this case, there are two possible questions: (1) How old is Mary? and (2) Who is young? Typically, p would be an answer to question 1 . Such a question is referred to as the default question, that is, the question that would normally be assumed unless explicitly stated to the contrary.

Assuming that the question is the first one, the meaning of $p$ would be represented as

$$
p \rightarrow \text { Age(Mary) is young }
$$

where Age(Mary) is the constrained variable; young is the constraining relation; and the constraint defines the possibility distribution of Age(Mary). If the membership function of young is defined as shown in figure 2 , then the same function defines the possibility distribution of Age(M ary). More specifically, if the grade of membership of, say, 25 in young is 0.8 , then the possibility that Mary is 25 given that Mary is young is 0.8 .

If the question is the second one, the meaning of $p$ would be represented as

$$
p \rightarrow \text { Person(young) isv Mary }
$$

in which the constrained variable is Person(young), and isv is a veristic constraint. Thus, if Mary is 25, and the grade of membership of 25 in young is 0.8 , then the verity (truth value) of the proposition "Mary is young" is 0.8 .

As a further example, consider the proposition p: Carol lives in a small city near San Francisco. Assume that the question is, What is the location of residence of Carol? Representation of the meaning of $p$ would proceed as follows:

Construct an explanatory database, ED, that consists of relations in terms of which the meaning of $p$ is defined (Zadeh 1986). Assume that the relations in ED are

$$
\begin{aligned}
\text { ED }= & \text { POPULATION }[\text { Name; Residence }+ \\
& \text { SMALL[City; } \mu]+ \\
& \text { NEAR[City1; City2; } \mu]
\end{aligned}
$$

In this case,

$$
\begin{aligned}
X & =\text { Residence }(\text { Carol }) \\
& \left.={ }_{\text {Residence }} \text { POPULATION[Name }=\text { Carol }\right]
\end{aligned}
$$

and

$$
\begin{aligned}
R= & \text { SMALL[City; } \mu] \cap \\
& \text { City } 1 \text { NEAR[City } 2=\text { San_Francisco }]
\end{aligned}
$$

In $R$, the first constituent is the fuzzy set of small cities, the second constituent is the fuzzy set of cities that are near San Francisco, and $\cap$ denotes the intersection of these sets. A left subscript denotes projection on the domain of the displayed variable.

An important concept that emerges from a synthesis of the concepts of CSNL and GCL is precisiated natural language (PNL). In essence, PNL is a subset of a natural language that consists of propositions that are precisiable through translation into GCL (figure 9). In this perspective, GCL plays the role of a precisiation language, with the understanding that precisiation of meaning is not coextensive with representation of meaning. For example, in the proposition "usually Robert returns from work at about 6 PM," a request for precisiation might be expressed as "I understand what you mean but could you be more precise about the meaning of 'usually' and 'about 6 PM'."

The importance of the concept of PNL derivesfrom the fact that by construction, GCL is maximally expressive because it incorporates all possible constraints and their combinations, modifications, and qualifications. It follows then that PNL is the largest subset of a natural language that admits precisiation. The high expressive power of PNL allows it to serve 


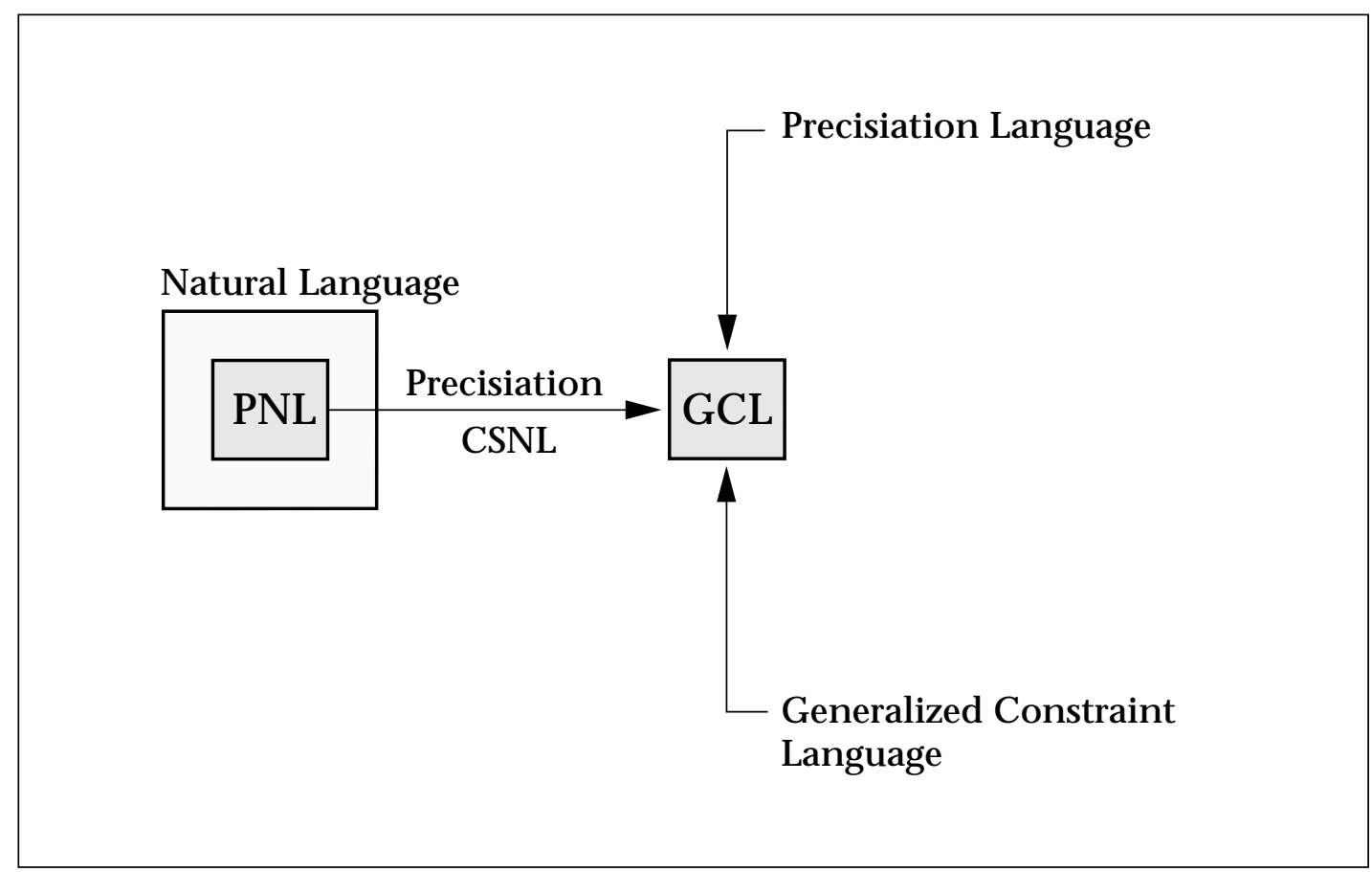

Figure 9. PNL as a Precisiated Subset of a N atural Language.

$\mathrm{PNL}$ - precisiated natural language. CSNL = constraint-centered semantics of natural languages.

as a powerful definition language, opening the door to (1) definition of new concepts and (2) redefinition of existing concepts in a more general setting. Simple examples of definitions are the concept of a smooth function and the concept of the usual, rather than expected, value of a random variable. Examples of PNL-based redefinition are stability, Pareto optimality, and statistical independence.

A question arises about what can be said about concepts that do not admit presiciation within the framework of PNL. An example of such a concept is causality. Another example is randomness. Such concepts will be said to be amorphic.

\section{Reasoning with Perceptions Based on Generalized Constraint Propagation}

In the computational theory of perceptions, perceptions play the role of carriers of information. Thus, reasoning with perceptions involves a process of arriving at answers to specified questions given a collection of perceptions that constitute the initial data set (IDS). As a simple example, assume that the IDS consists of perceptions

$\mathrm{p}_{1}$ : Most Swedes are tall.

$\mathrm{p}_{2}$ : Most Swedes are blond.

The question is, What fraction of Swedes are both tall and blond?

The generalized constraint propagation (GCP) is a process that involves successive application of a collection of rules that govern combination, modification, qualification, and propagation of generalized constraints.

One of the basic rules governing GCP is the compositional rule. In its generalized generic form, it is expressed as

$X$ isr $R$

$$
\frac{(X, Y) \text { iss } S}{Y \text { ist } T}
$$

In particular, for probabilistic constraints, it reduces to the familiar Bayesian rule for combination of probabilities

$X$ isp $P$

$\frac{\mathrm{Y} \mid \mathrm{X} \text { isp Q }}{\mathrm{Y} \text { isp } \mathrm{P} \bullet \mathrm{Q}}$

where $Y \mid X$ denotes $Y$ conditioned on $X$, and $\bullet$ is the convolution of the probability distribution of $X$ with the conditional probability distribution of $Y$ given $X$.

Similarly, in the case of possibilistic constraints, the compositional rule reads

$X$ is $R$

$$
\frac{(X, Y) \text { is } \subseteq}{Y \text { is } R \bullet S}
$$

where $\mathrm{R} \bullet \mathrm{S}$ is the composition of the possibility distribution of $X$ with the joint possibility
The high expressive power of PNL allows it to serve as a powerful definition language, opening the door to (1) definition of new concepts and (2) redefinition of existing concepts in a more general setting. 


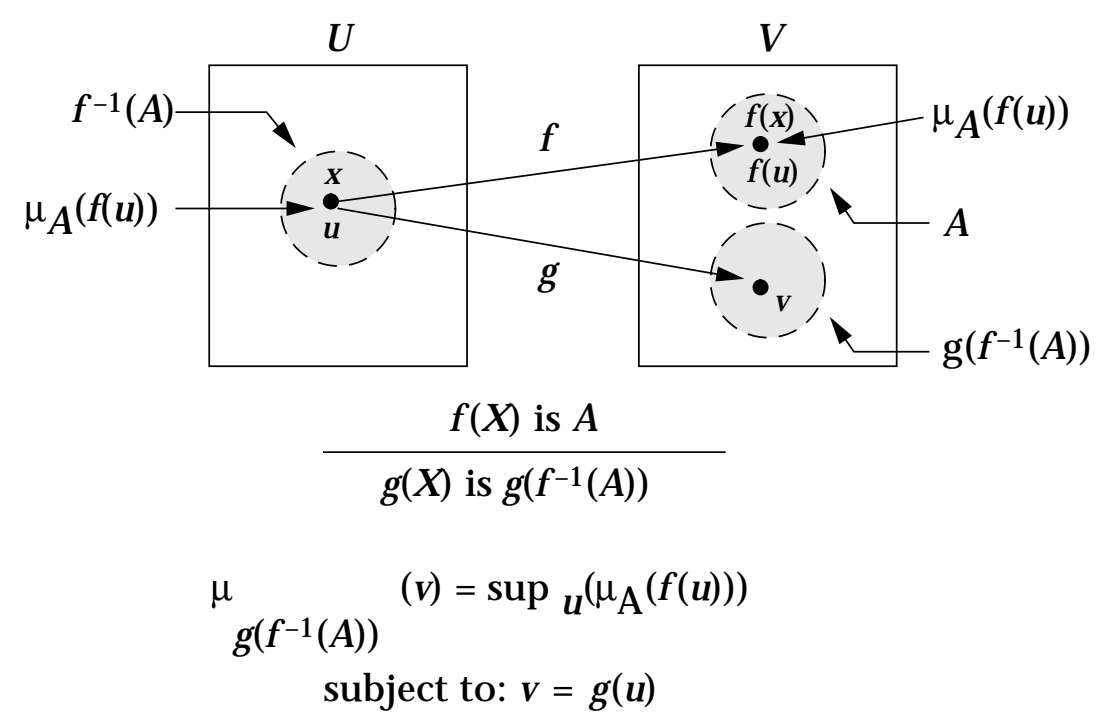

Figure 10. Schematic of the Generalized Extension Principle.

$f(X)$ is $A$ is a given constraint on $X$, and $g\left(f^{-1}(A)\right)$ is the induced constraint on $g(X)$. Computation of the induced constraint reduces to the solution of a variational problem.

distribution of $X$ and $Y$. For example, from the as perceptions

$\mathrm{p}_{1}:$ Mary is tall $\rightarrow$ Height(Mary) is tall

$\mathrm{p}_{2}$ : Chris is much taller than Mary $\rightarrow$

(Height(Chris), Height(Mary)) is much.taller we can infer the perception "Chris is T," where $\mathrm{T}=$ tall $\bullet$ much.taller. The $\bullet$ symbol is the operation of composition (Zadeh 1973). In this operation, conjunction and disjunction are usually assumed to be max and min, respectively. More generally, conjunction and disjunction can be, respectively, a t-norm and tconorm (Pedrycz and Gomide 1998).

Computational rules governing GCP become more complex when the constituent constraints are heterogeneous. For example, if $X$ is constrained probabilistically, and $(X, Y)$ is constrained possibilistically, as in

$X$ isp $P$

$$
\frac{(\mathrm{X}, \mathrm{Y}) \text { is } \mathrm{R}}{Y \text { isrs } T}
$$

then the constraint on $Y$ is of random set type. Such constraints play a central role in the Dempster-Shafer theory of evidence (Shafer 1976).

The principal rule of inference in the computational theory of perceptions is the generalized extension principle (Zadeh 1999) (figure 10). For possibilistic constraints, it can be expressed

$$
\frac{f(X) \text { is } R}{g(X) \text { is } g\left(f^{-1}(R)\right.}
$$

In this constraint-propagation rule, $f(X)$ is $R$ plays the role of an antecedent constraint that is an explicitation of a given perception or perceptions, $X$ is the constrained variable, $f$ is a given function, $R$ is a relation that constrains $f(x), g$ is a given function, and $f^{-1}(R)$ is the preimage of $R$.

In effect, $f(X)$ is $R$ is a general ized constraint that represents the information conveyed by antecedent perception(s), and $g(X)$ is $g\left(f^{-1}(R)\right)$ defines the induced general ized constraint on a specified function of $X$. As an elementary example, assume that the initial data set consists of two perceptions:

$\mathrm{p}_{1}$ : Most Swedes are tall.

$\mathrm{p}_{2}$ : Most Swedes are blond.

Explicitations of $p_{1}$ and $p_{2}$ can be expressed as

$\sum$ Count(tall.Swedes/Swedes) is most

$\sum$ Count(blond.Swedes/Swedes) is most where

$\sum$ Count(tall.Swedes/Swedes)

and

$\sum$ Count(blond.Swedes/Swedes)

represent, respectively, the proportions of tall 
Swedes and blond Swedes among Swedes. Application of the generalized extension principle leads to the following expression for the consequent constraint

¿Count(tall.and.blond.Swedes/Swedes)

is 2 most - 1

which retranslates into

(2most -1$)$ Swedes are tall and blond

In this expression, the membership functions of most and 2most - 1 are related, as shown in figure 4. Details of the derivation are shown in the box.

Note that in $\mathrm{p}_{1}$, tall is quantifiable by height, but blond in $p_{2}$ is not. When an attribute is not quantifiable, the grade of membership is associated directly with an individual rather than through an attribute. In many applications, especially in the real $m$ of control, el icitation of grades of membership is carried out through the use of automated learning techniques drawn from neurocomputing and evolutionary computing (Pedrycz and Gomide 1998).

In a general setting, application of the generalized extension principle transforms the problem of reasoning with perceptions into the problem of constrained maximization of the membership function of a variable that is constrained by a query. The examples considered earlier are simple instances of this process (Zadeh 1999).

What we see is that the machinery of the CTP bears little resemblance to the inference engine of predicate logic. At this juncture, CTP can be viewed as a direction-a direction that has a promise of enhancing the ability of Al to address problems in which the information that decisions are based on is perceptual in nature. This applies, in particular, to information about probabilities. More specifically, in most real-world settings, probabilities are not known precisely. What is known about them is, in the final analysis, based on perceptions rather than measurements.

\section{Concluding Remarks}

The CTP that is outlined in this article is not intended to replace traditional measurementbased methods. In effect, the theory is an additional tool that complements, rather than competes with, standard methods. The importance of CTP derives from the fact that much of human decision making and commonsense reasoning is, in reality, perception based. The principal features of CTP can be summarized as follows:

First, perceptions are assumed to be described by propositions drawn from a natur-
Computation with propositions that contain perceptual quantifiers such as most, several, and few requires a definition of cardinality of a fuzzy set to make it possible to answer a question such as, What fraction of Swedes are tall?

In fuzzy logic, cardinality is defined in two ways: (1) crisp cardinality, which issimpler; and (2) fuzzy cardinality. In this article, I consider only crisp cardinality.

If $A$ and $B$ are fuzzy sets in $U=\left\{u_{1}, \ldots, u_{N}\right.$ ) with respective grades of membership $\alpha_{1}, \ldots, \alpha_{\mathrm{N}}$ and $\beta_{1}, \ldots, \beta_{\mathrm{N}}$, then the sigma count of $\mathrm{A}$ is defined as

$$
\sum \operatorname{Count}(\mathrm{A})=\sum_{\mathrm{i}} \alpha_{\mathrm{i}} / \mathrm{u}_{\mathrm{i}}
$$

and the relative sigma count of elements of $A$ that are in $B$ is defined as

$$
\sum \operatorname{Count}(\mathrm{A} / \mathrm{B})=\frac{\sum_{\mathrm{i}} \alpha_{\mathrm{i}} \wedge \beta_{\mathrm{i}}}{\sum_{\mathrm{i}} \beta_{\mathrm{i}}}
$$

where $\wedge$ is min.

A basic identity involving sigma counts is the following:

$\sum \operatorname{Count}(A)+\sum \operatorname{Count}(B)=$

$\sum \operatorname{Count}(A \cap B)+\sum \operatorname{Count}(A \cup B)$

which is anal ogous to the basic identity for probabilities:

$$
\mathrm{P}(\mathrm{A})+\mathrm{P}(\mathrm{B})=\mathrm{P}(\mathrm{A} \cap \mathrm{B})+\mathrm{P}(\mathrm{A} \cup \mathrm{B})
$$

From the identity, it follows that

$$
\begin{aligned}
& \sum \operatorname{Count}(A)+\sum \operatorname{Count}(B)-N \leq \sum \operatorname{Count}(A \cap B) \leq \\
& \sum \operatorname{Count}(A) \wedge \sum \operatorname{Count}(B)
\end{aligned}
$$

Now, in the example under consideration, $A=$ tall.Swedes, $\mathrm{B}=$ blond.Swedes, and

$$
\begin{aligned}
& \frac{1}{\mathrm{~N}} \sum \text { Count(tall.Swedes / Swedes) is most } \\
& \frac{1}{\mathrm{~N}} \sum \text { Count(blond.Swedes / Swedes) is most }
\end{aligned}
$$

Substituting the given sigma counts into the inequalities, we obtain

$$
\left.2 \text { most }-1 \leq \frac{1}{\mathrm{~N}} \sum \text { Count(tall.Swedes } \cap \text { blond.Swedes }\right) \leq \text { most }
$$

Assuming that most is monotonic (figure 2), we can conclude that

$$
\frac{1}{\mathrm{~N}} \sum \text { Count(tall.Swedes } \cap \text { blond.Swedes) is } 2 \text { most }-1
$$

al language. Second, perceptions are assumed to be f-granular. Third, the meaning of a perception is expressed as a general ized constraint of the form $X$ isr $R$ or as a combination of such constraints. Translation is viewed as explicitation of $X, r$, and $R$. Fourth, reasoning or computation with perceptions involves a goal-directed propagation of general ized constraints from 
premises to conclusions. The principal rule that governs GCP is the generalized extension principle. Application of the generalized extension principle reduces, in general, to the solution of a variational problem with constraints that represent the sought-after information.

\section{Acknowledgment}

This article is dedicated to Claude Shannon, John McCarthy, and Marvin Minsky. This research was supported in part by Office of Naval Research (ONR) contract N00014-99-C-0298, National Aeronautics and Space Administration (NASA) contract NCC21006, NASA grant NAC2-117, ONR grant N00014-96-1-0056, ONR grant FDN0014991035, ARO grant DAAH 04-961-0341, and the BISC Program of the University of California at Berkeley.

\section{Bibliography}

Barsalou, L. W. 1999. Perceptual Symbol Systems. Behavioral and Brain Sciences 22:577-660.

Davis, E. 1990. Representations of Commonsense Knowledge. San Francisco, Calif.: Morgan Kaufmann.

Davis, E. 1987. Constraint Propagation with Interval Labels. Artificial Intelligence 32(3): 281-331.

de Kleer, J., and Bobrow, D. G. 1984. Qualitative Reasoning with Higher-Order Derivatives. In Proceedings of the Fourth National Conference on Artificial Intelligence. Menlo Park, Calif.: American Association for Artificial Intelligence.

Dubois, D., and Prade, H. 1996. Approximate and Commonsense Reasoning: From Theory to Practice. In Proceedings of the Foundations of Intelligent Systems, Ninth International Symposium, 19-33. Berlin: Springer-Verlag.

Dubois, D.; Fargier, H.; and Prade, H. 1994. Propagation and Satisfaction of Flexible Constraints. In Fuzzy Sets, Neural N etworks, and Soft Computing, eds. R. R. Yager and L. A. Zadeh, 166-187. New York: Von Nostrand Reinhold.

Forbus, K. D. 1984. Qualitative Process The ory. Artificial Intelligence 24(1): 85-168.

Geng, J. Z. 1995. Fuzzy CMAC Neural Networks. Journal of Intelligent and Fuzzy Systems 3(1): 87-102.

Kaufmann A., and Gupta, M. M. 1985. Introduction to Fuzzy Arithmetic: Theory and Applications. New York: Von Nostrand.

Kuipers, B. J. 1984. Qualitative Reasoning.
Cambridge, Mass.: MIT Press.

Lano, K. 1991. A Constraint-Based Fuzzy Inference System. In Proceedings of EPIA 91, Fifth Portuguese Conference on Artificial Intelligence, eds. P. Barahona, L. M. Pereira, and A. Porto, 45-59. Berlin: Springer-Verlag.

Lenat, D. B. 1995. cyc: A Large-Scale Investment in Knowledge Infrastructure. Communications of the ACM 38(11): 32-38.

McCarthy, J. 1990. Formalizing Common Sense, eds. V. Lifschitz and J. McCarthy. Norwood, N.J.: Ablex.

McCarthy, J., and Hayes, P. J. 1969. Some Philosophical Problems from the Standpoint of Artificial Intelligence. In Machine Intelligence 4, eds. B. Meltzer and D. Michie, 463-502. Edinburgh: Edinburgh University Press.

Mani, I., and Maybury, M. T., eds. 1999. Advances in Automatic Text Summarization. Cambridge, Mass.: MIT Press.

Mavrovouniotis, M. L., and StephanopouIoS, G. 1987. Reasoning with Orders of Magnitude and Approximate Relations. In Proceedings of the Sixth National Conference on Artificial Intelligence, 626-630. Menlo Park, Calif.: American Association for Artificial Intelligence.

Novak, V. 1991. Fuzzy Logic, Fuzzy Sets, and Natural Languages. International Journal of General Systems 20(1): 83-97.

Pedrycz, W., and Gomide, F. 1998. Introduction to Fuzzy Sets. Cambridge, Mass.: MIT Press.

Raiman, O. 1991. Order of Magnitude Reasoning. Artificial Intelligence 51(1): 11-38.

Sandewall, E. 1989. Combining Logic and Differential Equations for Describing RealWorld Systems. In Proceedings of the First International Conference on Principles of Knowledge Representation and Reasoning, 412-420. San Francisco, Calif.: Morgan Kaufmann.

Shafer, G. 1976. A Mathematical Theory of Evidence. Princeton, N.J.: Princeton University Press.

Struss, P. 1990. Problems of Interval-Based Qualitative Reasoning. In Qualitative Reasoning about Physical Systems, eds. D. Weld and J. de Kleer, 288-305. San Francisco, Calif.: Morgan Kaufmann.

Sun, R. 1994. Integrating Rules and Connectionism for Robust Commonsense Reasoning. New York: Wiley.

Vallee, R. 1995. Cognition et Systeme (Cognition and Systems). Paris: I'Interdisciplinaire Systeme(s).

Zadeh, L. A. 1999. From Computing with Numbers to Computing with Words-From Manipulation of Measurements to ManipuIation of Perceptions. IEEE Transactions on Circuits and Systems 45(1): 105-119.
Zadeh, L. A. 1997. Toward a Theory of Fuzzy Information Granulation and Its Centrality in Human Reasoning and Fuzzy Logic. Fuzzy Sets and Systems 90:111-127.

Zadeh, L. A. 1986. Outline of a Computational Approach to Meaning and Knowledge Representation Based on the Concept of a Generalized Assignment Statement. In Proceedings of the International Seminar on Artificial Intelligence and Man-M achine Systems, eds. M. Thoma and A. Wyner, 198-211. Heidelberg: Springer-Verlag.

Zadeh, L. A. 1973. Outline of a New Approach to the Analysis of Complex System and Decision Processes. IEEE Transactions on Systems, Man, and Cybernetics SMC3(1): 28-44.

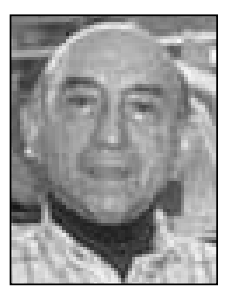

Lotfi A. Zadeh is a professor in the Graduate School at the University of California at Berkeley (UC Berkeley) and serves as director of the Berkeley Initiative in Soft Computing. He received his Ph.D. from Columbia University in 1949 and was promoted to the rank of professor in 1957. In 1959, he moved to UC Berkeley and served as chair of electrical engineering and computer science from 1963 to 1968 . His first paper on fuzzy sets was published in 1965, and since then, his research has focused on the development of fuzzy logic and its applications. Zadeh is a fellow of the American Association for Artificial Intelligence, the Association of Computing Machinery, and the Institute of Electrical and Electronics Engineers. He is a member of NAE and has received numerous awards in recognition of his development of fuzzy logic, among them the IEEE Medal of Honor and the ACM 2000 Allen Newell Award. His e-mail address is zadeh@cs.berkeley.edu. 